\title{
Isolated Maximal Surfaces in Spacetime
}

\author{
Dieter Brill* \\ Department of Physics and Astronomy, University of Maryland, College Park, Maryland 20742, USA \\ Frank Flaherty $\star \star$ \\ Department of Mathematics, Oregon State University, Corvallis, Oregon 97331, USA
}

\begin{abstract}
Maximal surfaces and their implications for the ambient spacetime are studied. Our methods exploit the interplay between contact of the volume functional and energy conditions. Essentially, we find that in closed universes, maximal surfaces are unique; they maximize volume; and they yield future and past singularities.
\end{abstract}

\section{Introduction}

The global properties of manifolds, in particular the types of extremal surfaces which they admit, have attracted much interest. In Riemannian manifolds, minimal surfaces-soap films and their generalizations to higher dimensionshave been the subject of numerous investigations [1]. The analogous surfaces in spacetimes, manifolds with Lorentz metric, are maximal surfaces. In asymptotically flat spaces, frequently a family of maximal surfaces folicates spacetime [2]. Such a foliation then gives a geometrically preferred time function. Maximal surfaces are also physically important for such questions as the analysis of the dynamics of the gravitational field or the analysis of the $n$-body problem in a gravitational field [3]. Abundant as they are in asymptotically flat spacetimes, maximal surfaces occur but rarely in spatially closed universes. Not surprisingly, their physical interpretation is also quite different. Namely, they typically describe the "turn around" epoch which separates the expansion from the recontraction phase. Today there are no theorems about the maximum expansion phase comparable in generality to the singularity theorems [5], which describe the universe's collapse. Here we initiate the study of the "turn around" epoch.

We consider three dimensional spacelike surfaces in spacetimes and the critical points of their volume functional. In Section II, we give the variational formulas for the first, second and higher variations of the volume away from a maximal

* Supported in part by the National Science Foundation under grant No. PHY 70-02077A03 and by the Humboldt Foundation

$\star \star$ Supported in part by the Sonderforschungsbereich (Theoretische Mathematik) of the University of Bonn 
surface. Section III applies the energy condition of general relativity to the question of uniqueness of maximal surfaces. The main theorem of the section shows that maximal Cauchy surfaces are essentially unique. Examples of closed spacetimes without any maximal surfaces are also given in this section. In Section IV we prove that in a vacuum spacetime maximal surfaces represent a strict maximum for the volume or are flat. Finally, in case a source satisfying an energy condition is present, maximal surfaces yield not only strict volume maxima but also entail both future and past singularities. In physical terms, then, if a maximal surface occurs in a universe, there is volume expansion and recontraction not only locally near the maximal surface, but the expansion must have begun at a singularity and the recontraction must proceed to a singularity.

The expressions in the body of the paper are written in coordinate-free notation to stress the geometric meaning. In the appendix we explain how to transcribe the expressions into coordinate notation more familiar in physics.

\section{Variational Formulas}

Let $M$ be a Lorentz manifold with Lorentz structure $\langle$, $\rangle$ and metric connection $D$. Let $S$ be a spacelike hypersurface (codimension 1) and let $S_{t}$ be a variation of $S$ through spacelike hypersurfaces, with $S_{0}=S$. Further let $d V_{t}$ be the volume element of $S_{t}$ in the induced Riemannian structure on $S_{t}$, and $V_{t}=\int_{S_{t}} d V_{t}$. We call $S_{t}$ a proper variation of $S$ iff $V_{t}$ is finite for any $t$ and the boundaries (if any) are fixed. Let $X$ be a vector field normal to the $S_{t}$ such that the local 1-parameter group $\phi_{t}$ generated by $X$ satisfies $\phi_{t}(S)=S_{t}$.

The covariant derivative in tangent direction $u$ of any vector field $Y$ normal to $S$ can be decomposed into normal and tangential components:

$$
D_{u} Y=\nabla_{u} Y-A^{Y}(u)
$$

$\nabla_{u} Y$ is the covariant derivative in the bundle of normal vectors to $S$, and $A^{Y}(u)$ is the second fundamental form on $S$. The operator $B$ defined by

$$
\langle B(u, v), Y\rangle=\left\langle A^{Y}(u), v\right\rangle
$$

gives rise by contraction to the mean curvature vector $H$, defined by

$$
H=\Sigma_{i} B\left(e_{i}, e_{i}\right)
$$

where $\left(e_{i}\right)$ is a local orthonormal frame field on $S$.

In terms of these quantities we can state the

Theorem 2.1 [4] If $S_{t}$ is a proper variation of $S$,

$$
\begin{aligned}
\dot{V}_{X}(0)= & \frac{d}{d t}\left(V_{t}\right)_{t=0}=-\int\langle H, X\rangle d V \\
\ddot{V}_{X}(0)= & \frac{d^{2}}{d t^{2}}\left(V_{t}\right)_{t=0}=\int(\langle\nabla X, \nabla X\rangle-\operatorname{Ricci}(X, X) \\
& \left.-\left\|A^{X}\right\|^{2}-\left\langle H, D_{X} X\right\rangle+\langle X, X\rangle\langle H, H\rangle\right) d V
\end{aligned}
$$


where $\left\|A^{X}\right\|^{2}$ is the norm squared of the $(1,1)$ tensor field $A^{X}$, and all integrals, here and in the following, are taken over the surface $S$.

Corollary 2.2. $S$ satisfies $\frac{d}{d t}\left(V_{t}\right)_{t=0}=0$ for all $X$ iff $H=0$. Such $S$ are called maximal surfaces.

Theorem 2.3. If $S_{t}$ is a proper variation of a totally geodesic ("time symmetric") hypersurface $S$, then $A^{X}=0=H$ for any $X$, and

$$
\frac{d^{3}}{d t^{3}}\left(V_{t}\right)_{t=0}=2 \int\left[\left\langle\nabla X, \nabla D_{X} X\right\rangle-\operatorname{Ricci}\left(X, D_{X} X\right)\right] d V
$$

Further, if $\nabla X=0$ on $S$, then

$$
\frac{d^{4}}{d t^{4}}\left(V_{t}\right)_{t=0}=2 \int\left[\left\langle\nabla D_{X} X, \nabla D_{X} X\right\rangle-\operatorname{Ricci}\left(D_{X} X, D_{X} X\right)-\left\|\mathscr{R}^{X, X}\right\|^{2}\right] d V
$$

where $\mathscr{R}^{X, X}(u, v)=\langle R(X, v) u, X\rangle, u, v$ tangent vectors, and \|\|$^{2}$ again indicates the norm squared of the $(0,2)$ tensor $\mathscr{R}^{X, X}$.

Proof. An outline of the Proof of 2.1-2.3 can be found in the Appendix.

Remark. With suitable interpretation the formulas of Section 2 apply not only to spacelike hypersurfaces but also to non-degenerate submanifolds.

\section{Uniqueness of Maximal Surfaces}

The well known static asymptotically flat spacetimes of general relativity provide examples of manifolds with a family of maximal surfaces $t=$ constant. Maximal surfaces in well known closed spacetimes, such as the Friedmann models [6], are much less frequent, unless the universe is static. Roughly, the spacetime either represents an ever expanding universe without any maximal spacelike surface, like the "open" Friedmann models; or the spacetime possesses just one maximal surface, the moment of maximum expansion, as in the typical "closed" Friedmann models.

In general if $M$ is a product $R \times S, S$ a Riemannian 3-manifold, with a metric

$$
d s^{2}=-d t^{2}+a(t)^{2} d \sigma^{2}
$$

it is easy to see that the spacelike sections $t=$ constant are totally umbillic, that is, the second fundamental form is a multiple of the surface's metric tensor

$$
\langle B(u, v), X\rangle=-X(\ln a)\langle u, v\rangle \text {. }
$$

Thus, for a spacelike section to be maximal, $X(a)=\partial_{t} a$ must vanish. The maximal surface in this case is a totally geodesic submanifold. In homogeneous universes it is generally true that each extremal of $a(t)$ corresponds to an extremal surface in this sense: suppose that a group of isometries acts on $M$ so that the orbits are compact spacelike hypersurfaces. Then any orbit which extremizes its volume compared to other nearby orbits is a homogeneous maximal surface. This fact follows from a theorem of Hsiang [7]. On the other hand if in (3.1) $\partial_{t} a$ is positive 
for all $t$, then the space contains no maximal spacelike surface whatever. To show this, we embedd any spacelike surface $S$ in the family $S_{t}$ obtained from $S$ by time translation. It is clear that for this variation $\dot{V}_{t}$ differs from zero. An example of a spacetime without maximal surfaces which satisfies the Einstein equations is the flat 3-torus of Kasner [8], where

$$
d s^{2}=-d t^{2}+t^{2} d x^{2}+d y^{2}+d z^{2} .
$$

In general spacetimes the extremal spacelike surfaces are not locally maximizing the volume, as they do for the expanding and contracting Friedmann universe. The equator of the deSitter space [9] is an example of a surface satisfying $H=0$, but which is a saddle point for the volume function. It is also an example of an extremal surface in a closed universe that forms part of a whole family of extremal surfaces - the "great spheres" of the deSitter universe (generalizations of the great circles on a sphere). These circumstances, however, cannot happen if the Ricci curvature is positive:

Theorem 3.1. If Ricci $(u, u)>0$ for future timelike $u$ and $S$ is an extremal hypersurface, then $\ddot{V}_{X}(0)<0$ for any normal vector field $X$ vanishing on $\partial S$.

Proof. $\ddot{V}_{X}(0)$ is given by Equation (2.1). By hypothesis Ricci is positive in timelike directions so that the integrand is positive.

This theorem shows that if the strong energy condition holds, a maximal surface in a closed spacetime cannot be part of a family of maximal surfaces. At this point we use the terminology maximal for extremal in the same way that minimal is used in Riemannian manifolds. Our next theorem shows that in fact there can be no other maximal surfaces anywhere in the domain of dependence of a given maximal spacelike surface. First we recall some definitions and propositions.

The notation $I^{+}(A, B)$ represents the set of all points in $B$ that can be joined to points in $A$ by a future timelike curve. The edge of an achronal set $S$ is the set of all $q$ in the closure of $S$ such that in every neighborhood $U$ of $q$ there is a $p$ in $I^{-}(q, U)$ and $r$ in $I^{+}(q, U)$ joinable by a timelike curve in $U$ missing $S$. Further an achronal set $S$ is called a partial Cauchy surface if its edge is empty.

Proposition 3.2. If $S$ is a partial Cauchy surface, the future domain of development of $S$ is globally hyperbolic.

Proof. A proof of this proposition can be found in the book of Hawking and Ellis $[5]$.

Theorem 3.3. Let $T$ be a maximal compact spacelike hypersurface contained in the future domain of development of $S$ where $S$ is a maximal partial Cauchy surface. If $\operatorname{Ricci}(u, u)>0$ for all future timelike $u$, then $T$ is contained in $S$.

Proof. If there is a point in $T$ which is not in $S$ then the function $f: p \rightarrow d(p, S)$ has a positive maximum as $p$ runs through $T$. Suppose the maximum occurs at $r$ in $T$. Then there is a $q$ in $S$ such that $f(r)=d(r, q)$. By global hyperbolicity of $D^{+}(S)$ 
there is a geodesic segment $c$ from $r$ to $q$ of length $d(r, q)=d(r, S)$, and $c$ must be orthogonal both to $T$ and $S$. Recall the second variation formula for arc length [10]:

$$
\begin{aligned}
\mathscr{L}_{u}^{\prime \prime}(0)= & \int_{0}^{b}\left(-\left\|D_{v} u\right\|^{2}+\langle R(u, V) V, u\rangle\right) d s \\
& +\langle B(u(0), u(0)), V(0)\rangle-\langle B(u(b), u(b)), V(b)\rangle .
\end{aligned}
$$

Here $u$ is a normal variational field along $c$ and $V$ is the tangent of $c$. Since $c$ maximizes the distance, $\mathscr{L}_{u}^{\prime \prime}(0) \leqq 0$ for all normal vector fields $u$. Consider vector fields along $c$, say $u_{1}, u_{2}, u_{3}$, obtained by parallel translation of an orthonormal basis in the tangent space at $q$. The resulting vectors $u_{1}(b), u_{2}(b), u_{3}(b)$ will form an orthonormal basis of the tangent space at $r$. Therefore we have

$$
\sum_{1 \leqq i \leqq 3} \mathscr{L}_{u_{\imath}}^{\prime \prime}(0)=\int_{0}^{b} \operatorname{Ricci}(V, V) d s
$$

since the trace of $B$ vanishes both at $q$ and at $r, S$ and $T$ being maximal. The fact that Ricci is positive yields $\mathscr{L}_{u_{j}}^{\prime \prime}(0)>0$ for some $j$. This contradicts the maximizing property of $c$. Therefore we must have $T$ as a subset of $S$.

We remark that the spirit of this proof goes back to Synge but the idea here comes from the work of Frankel [11]. Our theorems are also related to results, obtained by different methods, by Choquet-Bruhat [12]. We also remark that an entirely analogous theorem and analogous proof hold if $T$ and $S$ are hypersurfaces of a given constant mean curvature $H \neq 0$, rather than being maximal.

\section{Energy Conditions and Maximal Surfaces}

The assumption that space at one instant of time satisfies a Plateau problem or that the initial data on a spacelike hypersurface $S$ requires $S$ to be maximal is reasonable on physical and mathematical grounds. In this last section we shall discuss various implications arising from this assumption. The general question of existence will be the topic for later work [13].

A useful fact from the calculus of variations [14], employed in this section, is that if $\ddot{V}_{X}(0)<0$ for all normal fields $X$ then $S$ locally maximizes the volume function in the sense that for any proper variation $S_{t}$ of $S, V_{t}<V$ for sufficiently small $t$.

Theorem 4.1. Let $M$ satisfy the Einstein vacuum equations and suppose that $S$ is a closed maximal hypersurface. Then either $S$ locally maximizes volume or $S$ is a totally geodesic submanifold of $M$.

Proof. From Equation (2.1)

$$
\ddot{V}_{X}(0)=\int\left(\langle\nabla X, \nabla X\rangle-\left\|A^{X}\right\|^{2}\right) d V
$$

So either $\ddot{V}_{X}(0)<0$ for all normal vector fields $X$, in which case $S$ locally maximizes volume, or $\ddot{V}_{X}(0)=0$ for some $X$ not identically zero. Equation (4.1) then yields $\nabla X=0$ and $A^{X}=0$ on $S$. Hence $X$ is a normal field of constant length and $S$ is a totally geodesic submanifold. 
Remark. Since the hypersurface $S$ is orientable the existence of $X$ in the latter part of the theorem gives rise to an orientation of $M$ in which $M$ is time oriented.

Our next theorem uses one of the conclusions of Theorem 4.1 as a starting point. Later we shall synthesize both theorems.

Theorem 4.2. Suppose that $M$ is time oriented and satisfies the Einstein vacuum equations. Further let $S$ be a closed time symmetric hypersurface. Then either $S$ locally maximizes volume or $S$ is flat and hence the Cauchy development of $S$ is flat spacetime.

Proof. The proof examines the second, third, and fourth variations for a normal vector field $X$. Since

$$
\ddot{V}_{X}(0)=\int\langle\nabla X, \nabla X\rangle d V
$$

either $S$ locally maximizes volume in direction $X$ or $\nabla X=0$. In the latter case $X$ has constant length. From Equation (2.2)

$$
\dddot{V}_{X}(0)=2 \int\left(\left\langle\nabla X, \nabla D_{X} X\right\rangle-\operatorname{Ricci}\left(X, D_{X} X\right)\right) d V
$$

which vanishes because Ricci vanishes and $\nabla X=0$. Now we use Equation (2.3) to obtain

$$
\dddot{V}_{X}(0)=2 \int\left(\left\langle\nabla D_{X}, X, \nabla D_{X} X\right\rangle-\operatorname{Ricci}\left(D_{X} X, D_{X} X\right)-\left\|\mathscr{R}^{X, X}\right\|^{2}\right) d V .
$$

Thus either $\dddot{V}_{X}(0)<0$ and again $S$ locally maximizes volume in direction $X$ or $\mathscr{R}^{X, X}$ is zero on $S$. The hypotheses together with the vanishing of $\mathscr{R}^{X, X}$ imply that $S$ is Ricci flat and thus flat. As a result the Cauchy development $D(S)$ (of a flat, timesymmetric hypersurface) is flat spacetime. Combining Theorems 4.1 and 4.2 we obtain

Theorem 4.3. If $M$ is a time oriented spacetime that satisfies the Einstein vacuum equations and $S$ is a closed maximal hypersurface then $S$ locally maximizes volume or $S$ is flat and the Cauchy development of $S$ is flat spacetime.

To conclude this section we investigate the relationship between maximal hypersurfaces and geodesic completeness. First let us define the operator $L$

$$
L(X)=\nabla^{2} X+A^{2}(X)
$$

by

$$
\int\langle L(X), X\rangle d V=-\int\left(\langle\nabla X, \nabla X\rangle-\left\|A^{X}\right\|^{2}\right) d V
$$

where $X$ is a normal vector field to $S$. It is clear that $L$ is a strongly elliptic operator on the space of all normal vector fields to $S$ and as such possesses a minimum positive eigenvalue $\lambda_{1}$, such that $-\langle L X, X\rangle \leqq \lambda_{1}\langle X, X\rangle$ since $X$ is timelike.

Theorem 4.4. Suppose that $M$ satisfies the Einstein equations with cosmological constant $\lambda<\lambda_{1}$ coupled to a source which obeys the strong energy condition. Then $S$ locally maximizes volume and $M$ is timelike geodesically incomplete in the past as well as the future of $S$. 
Proof. The fact that the source obeys the strong energy condition implies that $\operatorname{Ricci}(X, X)-\lambda\langle X, X\rangle \geqq 0$.

for all timelike vector fields $X$. But

$$
\ddot{V}_{X}(0)=-\int(\langle L(X), X\rangle-\operatorname{Ricci}(X, X)) d V \leqq \int\left(\lambda_{1}-\lambda\right)\langle X, X\rangle d V .
$$

So $\ddot{V}_{X}(0)<0$ for all timelike normal $X$. Furthermore from Equation (A.6) we obtain

$$
\left\langle X, D_{X} H\right\rangle \leqq\left(\lambda_{1}-\lambda\right)\langle X, X\rangle<0 .
$$

Hence deforming $S$ for small positive $t$ results in hypersurfaces with negative mean curvature and deforming $S$ for small negative $t$ result in $S_{t}$ with positive mean curvature. In any case Hawking's theorem 4 of Chapter 8 [5] applies and $M$ is timelike incomplete to the future as well as the past of $S$.

Remark. From the Jordan-Brouwer separation theorem [15] $S$ separates $M$ into two components so that the hypersurfaces $S_{t}$ for positive $t$ lie in one component of $M-S$ and the $S_{t}$ for negative $t$ lie in the other component of $M-S$.

\section{Appendix}

In this appendix we rewrite the important equations of Section II in the notation used more frequently in physics [6].

Let $x^{i}$ be a coordinate system on $S$ and choose the coordinate basis $e_{i}=\partial_{i}$. The induced metric on $S$ then has the components $g_{i j}=\left\langle e_{i}, e_{j}\right\rangle$. Since $X$ is normal to $S$, the transformations $\phi_{t}$ can be used to generate a time orthogonal coordinate system in the neighborhood of $S$. The hypersurfaces $S_{t}$ are then described by $t=$ constant, and the deformation vector $X$ is given by $X=\partial_{t}$. The (positive) norm of $X$ is called the lapse function $N$,

$$
X=N U
$$

with $U$ a unit normal to $S_{t}$.

The covariant derivative in the normal bundle of any normal vector $X$ in direction $v$ is given by

$$
\nabla_{v} X=v(N) U=v^{i} N,_{i} U,
$$

and the second fundamental form $A^{X}$ has the components

$$
A_{i j}^{X}=\left\langle e_{i}, A^{X}\left(e_{j}\right)\right\rangle=-\left\langle e_{i}, D_{j} X\right\rangle=-N K_{i j}
$$

in terms of the usual extrinsic curvature tensor $K_{i j}=\left\langle e_{i}, D_{j} U\right\rangle$. Similarly, $B$ and $H$ can be expressed in terms of $K_{i j}$,

$$
\begin{aligned}
B\left(e_{i}, e_{j}\right) & =-K_{i j} U \\
H & =-g^{i j} K_{i j} U .
\end{aligned}
$$

The square of the norm of $A^{X}$ is

$$
\left\|A^{X}\right\|^{2}=N^{2} K_{i j} K^{i j}
$$


and the operator used in Theorem 4.4 becomes

$$
A^{2}(X)=N K_{i j} K^{i j}\langle U, U\rangle U .
$$

(The negative sign in some of these formulas is dictated by the desire to agree with the notation of Simons [16] in the invariant formulation, and with the notation of MTW [6] in the coordinate formulation.)

We also have

$$
\operatorname{Ricci}(X, X)=R_{00}=g^{i j} \mathscr{R}^{X, X}\left(e_{i}, e_{j}\right),
$$

and

$$
\mathscr{R}^{X, X}\left(e_{i}, e_{j}\right)=R_{0 i 0 j}=N^{2}\langle U, U\rangle\left(R_{i j}+K_{i l} K_{j}^{l}-K_{i j} K_{l}^{l}-{ }^{3} R_{i j}\right),
$$

with $R_{\alpha \beta \gamma \delta}, R_{\mu \nu}$, and ${ }^{3} R_{i j}$ denoting the components of the Riemann tensor of $M$, the Ricci tensor of $M$, and the Ricci tensor of $S$, respectively. The norm squared,

$$
\left\|\mathscr{R}^{X, X}\right\|^{2}=g^{i k} g^{j l} R_{0 i 0 j} R_{0 k 0 l}
$$

takes a simple form for a totally geodesic $S\left(K_{i j}=0\right)$ in the vacuum case $\left(R_{\mu \nu}=0\right)$,

$$
\left\|\mathscr{R}^{X, X}\right\|^{2}={ }^{3} R_{i j}{ }^{3} R^{i j} .
$$

The formulas of the Theorems can now be easily transcribed into this notation. For example, Theorem 2.1 says (with $\dot{N}=\partial_{t} N$ )

$$
\begin{aligned}
d V / d t= & \int g^{i j} K_{i j} N d V \\
d^{2} V / d t^{2}= & \int\left[g^{i j} N,{ }_{i} N,{ }_{j}\langle U, U\rangle-R_{00}-K_{i j} K^{i j} N^{2}\right. \\
& \left.+K_{i}^{i} N \dot{N}+\left(K_{i}^{i}\right)^{2} N^{2}\right] d V .
\end{aligned}
$$

Note also that the operator $L$ introduced in Section IV has the coordinate expression, in terms of the Laplacian $\Delta$ of $S$,

$$
L(X)=\left(\Delta N+K_{i j} K^{i j} N\langle U, U\rangle\right) U .
$$

The proof of Theorems 2.1-2.3 in the coordinate version (A.2), (A.3) etc. is obtained by repeated application of the expressions for the normal derivatives of the metric and extrinsic curvature components,

$$
\begin{aligned}
\partial_{t} g_{i j} & =2 K_{i j} N \\
\partial_{t} K_{i j} & =K_{i l} K_{j}^{l} N-\mathscr{R}^{X, X}\left(e_{i}, e_{j}\right) N^{-1}-N_{; i j}\langle U, U\rangle .
\end{aligned}
$$

Equation (A.4) follows from the definition of $K_{i j}$,

$$
\partial_{t} g_{i j}=X\left(\left\langle e_{i}, e_{j}\right\rangle\right)=2\left\langle D_{X} e_{i}, e_{j}\right\rangle=2 N\left\langle D_{i} U, e_{j}\right\rangle
$$

Similarly (A.5) results upon working out the expression

$$
X\left(K_{i j}\right)=\left\langle D_{X} e_{i}, D_{j} U\right\rangle+\left\langle e_{i}, D_{j} D_{X} U\right\rangle+\left\langle e_{i}, R\left(X, e_{j}\right) U\right\rangle,
$$

noting that $D_{X} U=-\langle U, U\rangle g^{k l} N_{, k} e_{l}$ and that, in this Appendix, $\left[X, e_{i}\right]=$ $\left[\partial_{t}, \partial_{i}\right]=0$. 
Finally, the equation necessary for Theorem 4.4 follows from (A.1)-(A.3),

$$
\begin{aligned}
\left\langle X, D_{X} H\right\rangle & =-N\langle U, U\rangle \partial_{t}\left(g^{i j} K_{i j}\right) \\
& =(\Delta N) N+K_{i j} K^{i j} N^{2}\langle U, U\rangle+R_{00}\langle U, U\rangle \\
& =[\langle L(X), X\rangle+\operatorname{Ricci}(X, X)]\langle U, U\rangle
\end{aligned}
$$

and is equivalent to the familiar "Raychaudhuri equation"

$$
-\partial_{t}\left(K_{i}^{i}\right)=-\Delta N+K_{i j} K^{i j} N+R_{\hat{0} \hat{0}} N .
$$

\section{References}

1. See, for example, Nitsche, J.C.C.: Vorlesungen über Minimalflächen. Berlin-Heidelberg-New York: Springer 1975

2. For an example, see, Reinhart, B. L.: J. Math. Phys. 14, 719 (1973) For existence proof see, Choquet-Bruhat, Y.: C. R. Acad. Sci. (Paris) 280, 169 (1975);

Cantor, M., Fischer, A., Marsden,J., O’Murchadha, N., York, J.: Commun. math. Phys. 49, 187 (1976)

3. Arnowitt, R., Deser, S., Misner,C.W.: In: Gravitation (ed. L.Witten). New York: Wiley 1962 Brill, D., Deser, S.: Ann. Phys. (NY) 50, 548 (1968);

York, J., O'Murchadha, N.: J. Math. Phys. 14, 1551 (1973);

Schücking, E.: Proceedings of Marcel Grossman Symposium, to appear

4. See also Frankel, T.: Bull. Amer. Math. Soc. 81, 579 (1975)

5. See, for example, Hawking, S., Ellis, G.: The large scale structure of space-time, Cambridge: University Press 1973

6. See, for example, Misner, C.W., Thorne,K., Wheeler, J.A.: Gravitation. San Francisco: W.H.Freeman and Company 1973

7. Hsiang, W. Y.: Proc. Nat. Acad. Sci. (USA) 56, 5 (1966)

8. Kasner, E. : Amer. J. Math. 43, 217 (1921)

9. See, for example, Schrödinger,E.: Expanding Universes. London: Cambridge University Press 1956

10. See Gromoll,D., Klingenberg,W., Meyer,W.: Riemannsche Geometrie im Großen. BerlinHeidelberg-New York: Springer 1968

Hicks, N.: Notes on Differential Geometry. Princeton: D. van Nostrand 1965

11. Frankel, T.: Pacific J. Math. 11, 165 (1961)

12. Choquet-Bruhat, Y.: C.R. Acad. Sci. (Paris) 281, 577 (1975)

13. Also see Choquet-Bruhat, Y.: C.R. Acad. Sci. (Paris) 280, 169 (1975). For an existence proof in the case of a periodic closed space-time, see Avez, A.: Ann. Inst. Fourier 13, 105 (1963)

14. See, for example, Corollary 3.6.1 of Simons, J.: Ann. Math. (USA) 88, 62 (1968)

15. For example, see Spanier, E. H.: Algebraic topology. New York: McGraw-Hill Publishing Co. 1966

16. Simons, J.: Ann. Math. (USA) 88, 62 (1968)

Communicated by J. Ehlers

Received January 8, 1976 
